\title{
New programs for determining the main parameters of open clusters
}

\author{
M.E. Popova ${ }^{\star}$ \\ Astronomical Observatory, Ural Federal University, 51 Lenin Street, Yekaterinburg 620000, Russia
}

Received 2013 Apr 3, accepted 2013 Jun 7

Published online 2013 Oct 1

Key words open clusters and associations: general - techniques: photometric

A set of computer programs for the determination of the main parameters of open clusters, such as color excess, distance, and age, is developed. The set consists of two units. The first unit is designed to search for open clusters in wide fields using $J H K_{\mathrm{s}}$-photometry from various catalogs (2MASS, UKIDSS, VISTA) by identifying areas of high stellar density. The second unit determines the parameters of open clusters by fitting photometric diagrams. This is the unit described in the paper. Probable cluster members are identified by the proximity to the basic sequences on the $J-(J-H)$ and $Q$ $(J-H)$ diagrams. The color excess is determined by minimizing the deviations of $Q$ values on the $Q-(J-H)$ diagram. Ages and distance moduli are determined by comparing the color-magnitude diagrams with theoretical isochrones.

(c) 2013 WILEY-VCH Verlag GmbH \& Co. KGaA, Weinheim

\section{Introduction}

A number of new all-sky photometric catalogs have appeared lately. Hence the need has arisen for simultaneous search for and analysis of new star clusters. Such a procedure would make it possible (a) to decide whether a particular cluster really exists, and (b) to search for new clusters and derive homogeneous estimates of cluster parameters. It is therefore necessary to develop a software set (see, e.g., Loktin, Gerasimenko \& Malysheva 2001) focusing on the search for new clusters combined with simultaneous determination of their main parameters (color excess, distance and age).

The search for clusters is carried out in wide fields ( $2^{\circ} \times 2^{\circ}$ with a $20 \%$ overlap) using $J H K_{\text {s }}$-photometry from a number of catalogs (2MASS, UKIDSS etc.). The program identifies areas of high stellar density via waveletsmoothing (see, e.g., Frick et al. 2001) and by seeking areas where stellar density distribution resembles that of a typical star cluster. The first unit of the program produces two data sets: that of the cluster area and that of the field area in close vicinity to the open cluster. Here we discuss the second unit developed in order to estimate cluster parameters.

\section{Identification of cluster members}

Probable cluster members are identified in photometric diagrams using weight factors characterizing the cluster membership probabilities of stars.

The first weight factor $P_{1}$ takes into account the photometric errors $\left(\sigma J^{2}, \sigma H^{2}, \sigma K^{2}\right)$ according to

$$
P_{1}=\frac{0.1}{\sigma J^{2}+\sigma H^{2}+\sigma K^{2}+0.01} \text {. }
$$

\footnotetext{
^ Corresponding author: Maria.Popova@usu.ru
}

The second weight factor $P_{2}$ is defined via the $J$ $(J-H)$ diagram. We compute star densities on the photometric diagrams of the open-cluster and field regions, each subdivided into many pixel bins (with the bin sizes equal to $0.5^{\mathrm{m}}$ in $J$ and $0.05^{\mathrm{m}}$ in $\left.(J-H)\right)$. We then subtract the density distribution of the field region from that of the cluster region (after normalizing them to the respective sky areas) and smooth the resulting difference via a digital filter. The computed densities are the second weight factor on the smoothed diagram (see Fig. 1).

We define the third weight factor $P_{3}$ by analogy with the second weight factor, but using the $Q-(J-H)$ diagram instead of the $J-(J-H)$ diagram. The parameter $Q$ is equal to $Q=(J-H)-1.95(H-K)$ (Bessel \& Brett 1988). We converted Ks values to standard magnitudes using the transformation $K=K_{\mathrm{s}}+0.044$ (Sarajedini et al. 2004). The bin sizes are equal to $0.05^{\mathrm{m}}$ in $(J-H)$ and $0.05^{\mathrm{m}}$ in $Q$. The weights of stars lying outside the $-0.1^{\mathrm{m}} \ldots+0.6^{\mathrm{m}}$ interval in $Q$ are additionally suppressed significantly. This interval is specific for most of the stars. The computed densities on the smoothed diagram give the third weight factor (see Fig. 2).

The fourth weight factor $P_{4}$ is inversely proportional to the deviation of the star's proper motion from the mean proper motion of the cluster. The fifth and sixth weight factors $P_{5}$ and $P_{6}$ are inversely proportional to the deviation of star's position from the line of unreddened stars in the $Q$ $(J-H)$ diagram and from the isochrone in the HR diagram, respectively.

The final weight of each star in the cluster region (which measures the cluster membership probability for the star considered) is given by the following formula:

$P=\left(P_{1} \cdot P_{2} \cdot P_{3} \cdot P_{4} \cdot P_{5} \cdot P_{6}\right)^{1 / 6}$.

We then remove stars with low weights from the sample (see Fig. 3). 


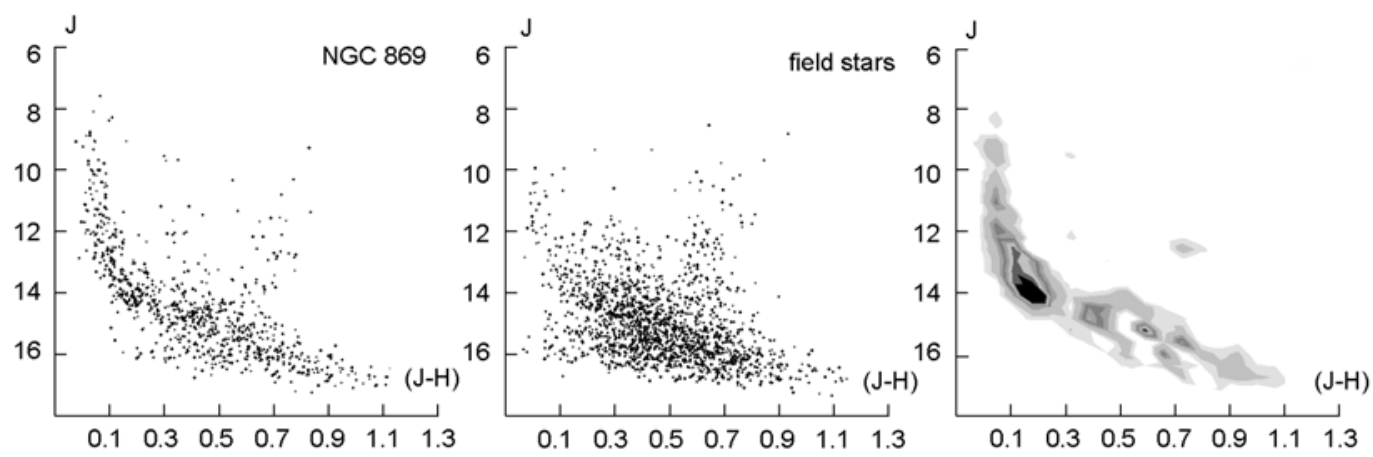

Fig. 1 The $J-(J-H)$ diagram for the NGC 869 region (left), field area (middle), and the smoothed diagram (right).
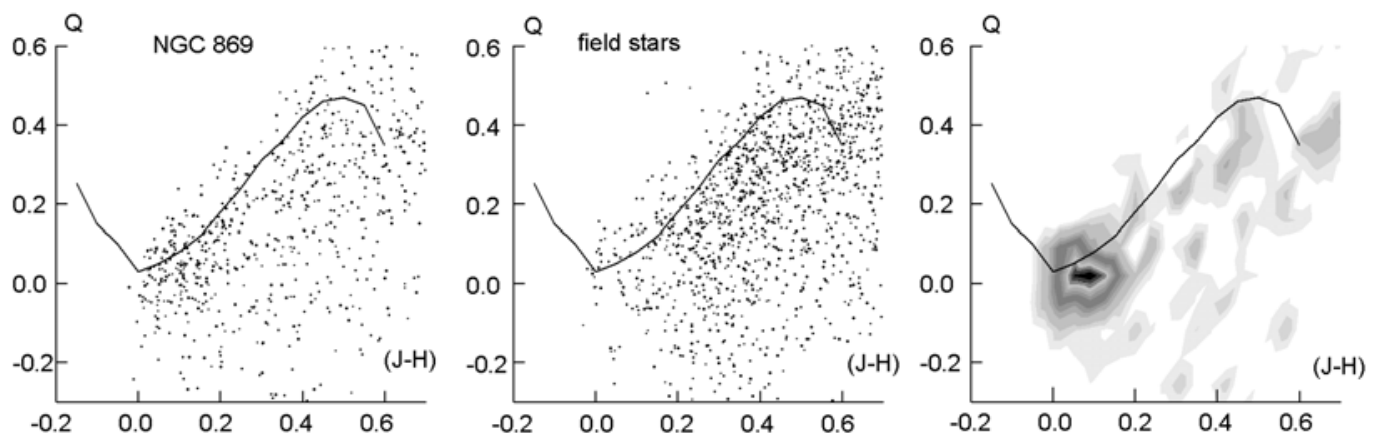

Fig. 2 The $Q-(J-H)$ diagram for the NGC 869 region (left), field area (middle), and the smoothed diagram (right). The solid line shows the locus of unreddened stars based on the data for nearby open clusters.
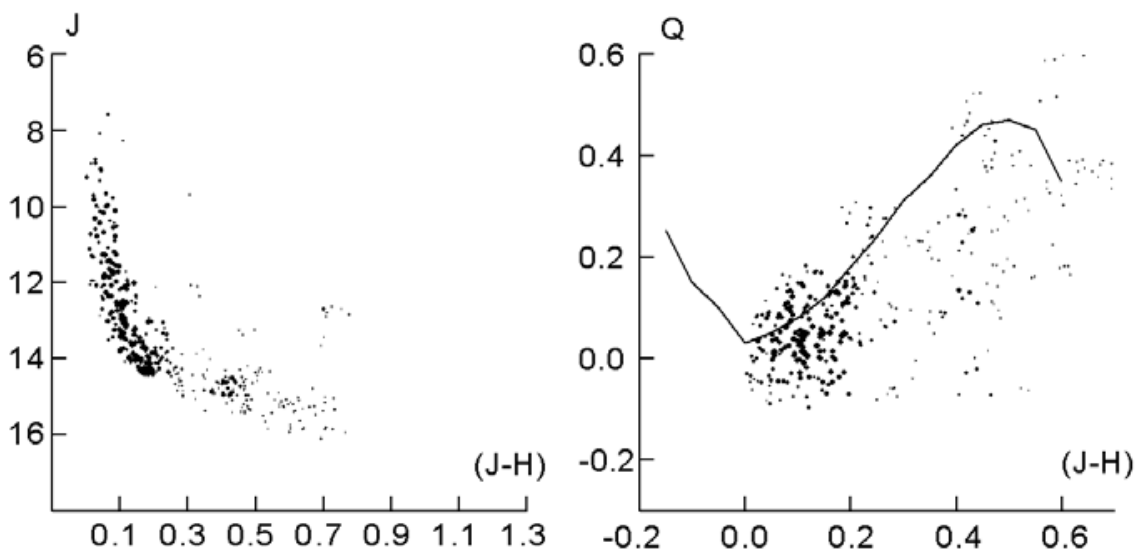

Fig. 3 The $J-(J-H)($ left $)$ and $Q-(J-H)$ diagrams (right) for the NGC,869 region. Circle diameters are proportional the calculated individual stellar weights.

\section{Determination of cluster parameters}

We determine the parameters of open clusters by fitting the $J-(J-H)$ and $Q-(J-H)$ photometric diagrams. The parameter $Q$ is equal to $Q=(J-H)-1.95(H-K)$ (Bessel $\&$ Brett 1988). We convert $K_{s}$ values to standard magnitudes using the transformation $K=K_{\mathrm{s}}+0.044$ (Sarajedini et al. 2004).

We determine the color excess by shifting star positions along the $(J-H)$ axis in the $Q-(J-H)$ diagram so as to minimize the sum of small powers of deviations of $Q$ values from the line of unreddened stars. The sum of deviations, ss, is calculated by the following formula:

$\mathrm{ss}=\sum_{i=1}^{n}\left(P_{i}\left|Q_{i}-Q_{0}\right|^{\alpha}\right)$

where $\alpha=50 / n$ for clusters with $n>50$ stars, or else $\alpha=2 . P_{i}$ is the weight of each star, $Q_{i}$ its $Q$ parameter, $Q_{0}$ the $Q$ parameter of an unreddened star having the same color index. Stars are shifted in $0.01^{\mathrm{m}}$ increments along the 


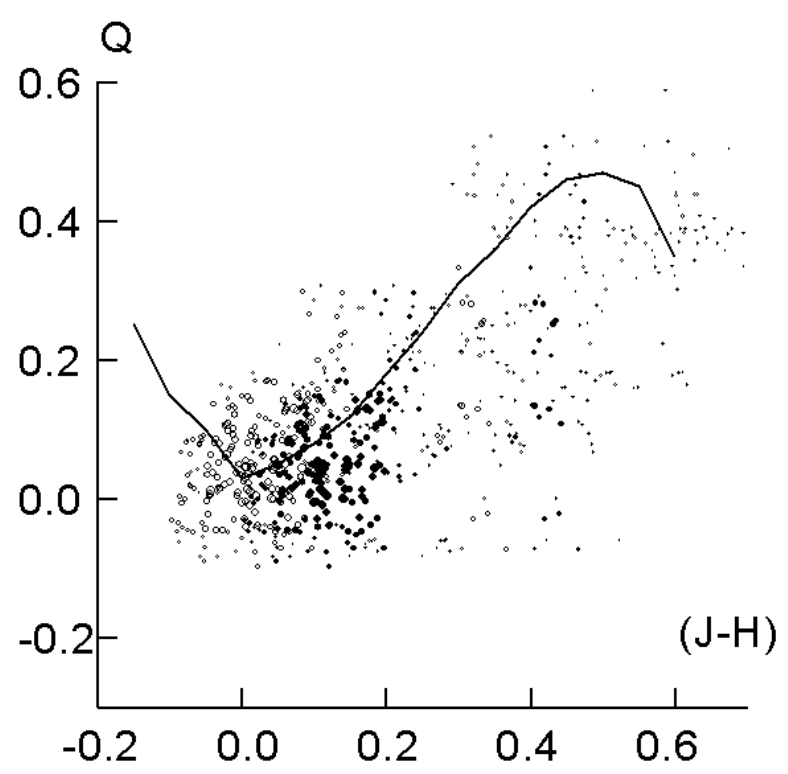

Fig. 4 The positions of NGC 869 stars in the $Q-(J-H)$ diagram (black circles) and the positions of the same stars shifted by their color excess (open circles).

$(J-H)$. We estimate the $(J-H)$ color excess of NGC 869 to be $0.10^{\mathrm{m}}$ when computed based 2MASS data (Skrutskie et al. 2006) (see Fig. 4).

After the correction for color excess we determine the distance moduli and ages of clusters by shifting star positions along the $J$ axis in the $J-(J-H)$ diagram so as to achieve the best fit of extinction-corrected magnitudes and color indices with one of the isochrones adopted from Girardi et al. (2003). The best-fit isochrone is determined by minimizing the sum of small powers of deviations of $(J-H)$ color indices (see Fig. 5). Stars are shifted in $0.25^{\mathrm{m}}$ increments along the $J$ line. The deviation of the lower part of the cluster main sequence from ZAMS can be explained by the small age of the cluster.

To test the inferred parameters, we computed the color excesses for each star by the following formula:

$E_{(J-H)_{i}}=(J-H)_{i}+0.03-1.18 Q_{i}$

(A.V. Loktin, this volume). The weighted average of the color excess is $0.12^{\mathrm{m}}$ and it differs by only $0.02^{\mathrm{m}}$ from the estimate obtained above.

We calculated the standard deviations $\sigma$ of the distance modulus, color excess, and age estimates for NGC 869 obtained using four different field areas around the cluster: $\sigma(\lg T)=0.15, \sigma\left(J_{0}-M_{J}\right)=0.06^{\mathrm{m}}$, and $\sigma\left(E_{(J-H)}\right)=$ $0.01^{\mathrm{m}}$. Hence the choice of the field area has practically no effect on the estimated open clusters parameters.

\section{Conclusions}

Figure 6 shows the color-magnitude and $Q$-diagrams for some clusters constructed using the program described

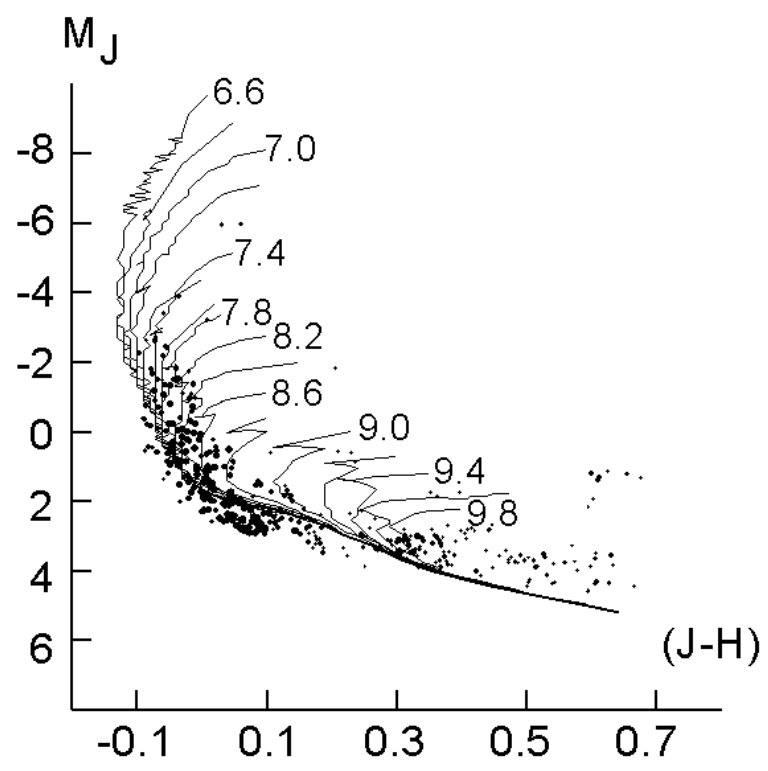

Fig. 5 The color-magnitude diagram for NGC 869. Heliocentric distance and age of the cluster are $=2.00 \mathrm{kpc}$ and $\lg T=7.4$, respectively.

above. Positions of faint stars $\left(J>15^{\mathrm{m}}\right)$ on the colormagnitude diagrams based on 2MASS data are of no practical use, because 2MASS photometric errors become very large at faint magnitudes. The results depend only slightly on the choice of the field area. Only the age estimate is sensitive to this choice, showing that this parameter is generally not reliably determinable. The analyses of some open clusters considered here demonstrate that the methods used in the program allow probable cluster members to be identified with satisfactory quality. The limited photometric accuracy for faint stars in the 2MASS catalogue restricts the studies of distant clusters. Deeper surveys such as UKIDSS, VISTA are expected to allow homogeneous estimates to be obtained for distant open clusters.

Acknowledgements. This work was supported by the Russian federal task program "Research and Operations on Priority Directions of Development of the Science and Technology Complex of Russia for 2007-2013" (contract 14.518.11.7064).

\section{References}

Bessel, M., \& Brett, J. 1988, PASP, 100, 1134

Frick, P., Beck, R., Berkhuijsen, E. M., \& Patrickeyevet, I. 2001, MNRAS, 327, 1145

Girardi, L., Bertelli, G., Bressan, A., et al. 2003, Mem. Soc. Astron. Ital., 74, 474

Loktin, A.V., Gerasimenko, T.P., \& Malysheva, L. K. 2001, Astronomical and Astrophysical Transactions, 20, 607

Sarajedini, A., Brandt, K., Grocholski, A. J., \& Tiede, G. P. 2004, AJ, 127, 991

Skrutskie, M. F., Cutri, R. M., Stiening, R., et al. 2006, AJ, 131, 1163 

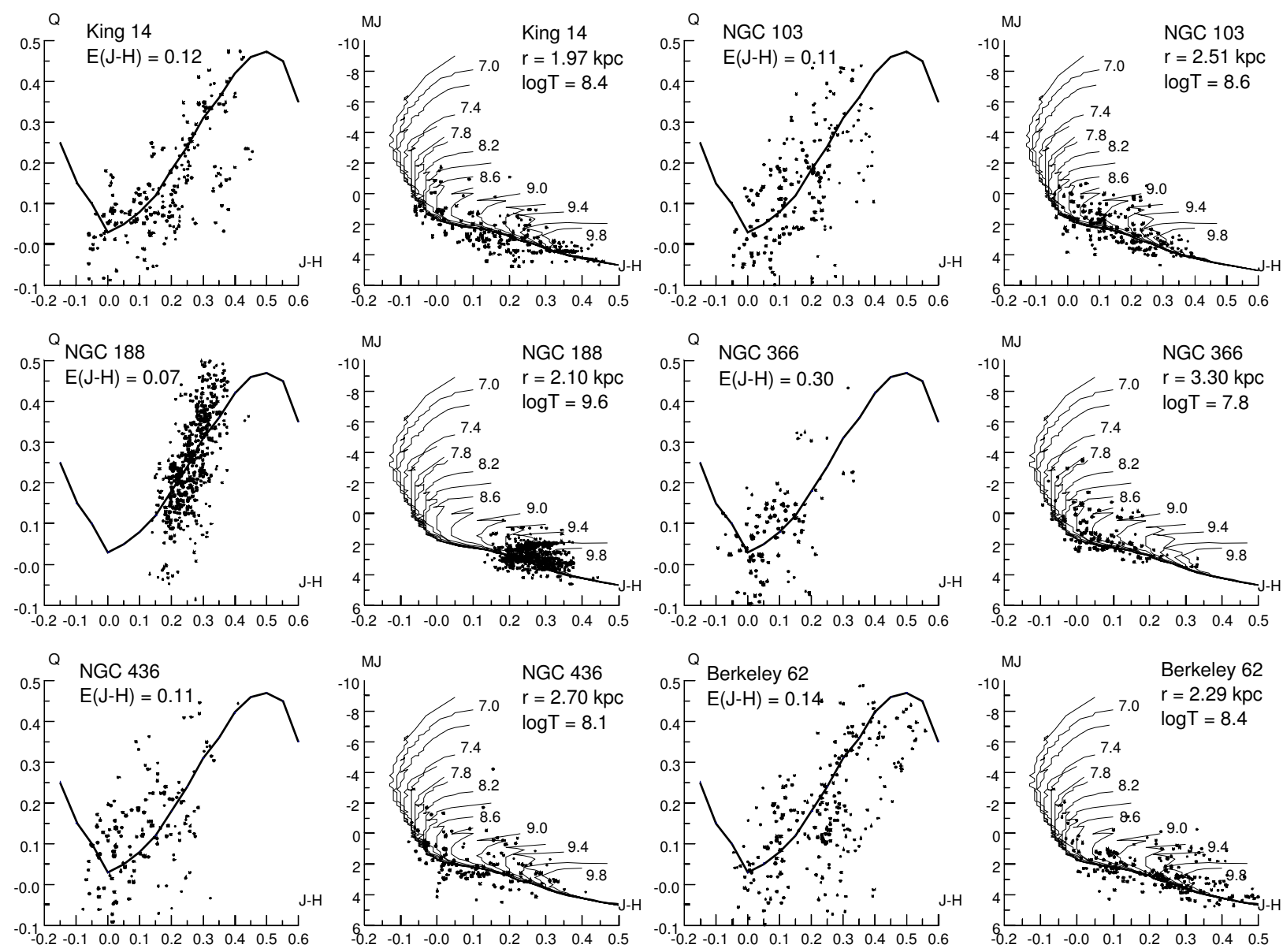

Fig. 6 The color-magnitude and $Q$-diagrams for some open clusters. 